\section{REFERENCES}

1 Bisogno G, Ferrari A, Bergeron C, et al. The IVADo regimen - a pilot study with ifosfamide, vincristine, actinomycin D, and doxorubicin in children with metastatic soft tissue sarcoma. Cancer 2005; 103: 1719-1724.

2 Kliem V, Bugge M, Leimenstoll K, et al. Pulmonary blastoma - a rare tumour. Clin Investig 1992; 70: 927-931.

3 Barret NR, Barnard WG. Some unusual thoracic tumours. Br J Surg 1945; 32: 447-457.

4 Koss M, Travis W, Moran C. Pulmonary sarcomas, blastomas, carcinosarcomas and teratomas. In: Hasleton PS, ed. Spencer's Histopathology of the Lung. 5th Edn. New York, McGraw Hill, 1996; pp. 1092-1100.

5 Priest JR, Hill DA, Williams GM, et al. Type I pleuropulmonary blastoma; a report from the International Pleuropulmonary Blastoma Registry. J Clin Oncol 2006; 24: 4492-4498.

6 Koss MN, Hochholzer L, O'Leary T. Pulmonary blastomas. Cancer 1991; 67: 2368-2381.

7 Zaidi A, Zamvar V, Macbeth F, et al. Pulmonary blastoma; medium-term results from a regional center. Ann Thorac Surg 2002; 73: 1572-1575.
8 Nakatani Y, Masudo K, Nozawa A, et al. Biotin-rich optically clear nuclei express estrogen receptor beta; tumors with morules may develop under the influence of estrogen and aberrant beta-catenin expression. Human Pathol 2004; 35: 869-874.

9 Esper A, Force S, Gal A, et al. A 36-year old woman with hemoptysis and a lung mass 3 months after delivery. Chest 2006; 130: 1620-1623.

10 Cutler CS, Michel RP, Yassa M, et al. Pulmonary blastoma; case report of a patient with a 7-year remission and review of chemotherapy experience in the world literature. Cancer 1998; 82: 462-467.

11 Barson AJ, Jones AW, Lodge KV. Pulmonary blastoma. J Clin Pathol 1968; 21: 480-485.

12 Kouvaris JR, Gogou PV, Papacharalampous XN, et al. Solitary brain metastasis from classic biphasic pulmonary blastoma; a case report and review of the literature. Onkologie 2006; 29: 568-570.

13 Zavala-Alarcon E, Sudhakar S, Gonzales LR, et al. Extension of pulmonary blastoma into the left atrium. Mayo Clin Proc 2001; 76: 657-660.

DOI: $10.1183 / 09031936.00187210$

\title{
Tuberculosis in the elderly in Germany
}

\section{To the Editors:}

Germany has a low tuberculosis (TB) incidence with a decreasing trend [1].

However, the proportion of TB patients aged $\geqslant 60 \mathrm{yrs}$ is increasing (1976: 26.5\%; 1986: 30.9\%; 1996: 33.7\%; 2006: 34.8\%. 1976 and 1986 figures are for the Federal Republic of Germany only), reflecting demographic changes. Age-related factors not only increase the risk of $\mathrm{TB}$ reactivation but also enhance susceptibility to TB infection, abetting outbreaks, e.g. in nursing homes $[2,3]$. To optimise healthcare services and TB control in this subpopulation of growing impact, detailed knowledge of the epidemiological features of TB in the elderly is needed.

National German TB notification data from 2002 to 2006 were analysed as a pooled 5-yr data set stratified into two age groups: TB patients 15-59 yrs (referred to as "younger adults") and those aged $\geqslant 60$ yrs ("the elderly"). For both groups, proportions of TB cases with reference to demographic factors, affected organs, drug resistance, case finding and treatment outcome were determined. Mortality (based on treatment outcome notification data), TB incidence, and age-specific TB incidence in 5-yr strata were calculated based on population data from regional statistical offices (time averaged 2002-2006). As the country of birth was unknown for the general population, origin-related TB incidence was derived from citizenship information. Proportion (\%) and incidence (cases per 100,000 population) were compared between both groups using the Z-test. Odds ratios (ORs), 95\% confidence intervals (CIs) and corresponding p-values were specified. Data were collected using the electronic reporting system SurvNet@rki (Robert Koch Institute, Berlin, Germany) and analysed using SPSS version 15 (SPSS Inc., Chicago, IL, USA), Excel (Microsoft Corporation, Redwood, CA, USA) and Stata version 11.0 (StataCorp LP, College Station, TX, USA).
A total of 31,459 TB cases aged $\geqslant 15$ yrs were identified. Details of the study set are presented in table 1 .

The proportion of elderly patients $(36.0 \%)$ remained stable over the study period. The elderly differed from the younger TB cases, first of all in patient origin: $45.0 \%$ of the younger TB cases had a foreign citizenship and $56.6 \%$ were born abroad, versus $12.0 \%$ and $23.1 \%$, respectively, of the elderly patients.

Proportions of pulmonary TB (PTB), which was the most common form, and of extrapulmonary TB (EPTB) were almost similar in the two age groups. However, the EPTB forms differed: genitourinary TB was more often diagnosed among the elderly, especially in males, while extrathoracic lymph node TB was seen less frequently. Among PTB patients, both culturepositivity rate and positive microscopy results were seen significantly less frequently in the elderly (76.2 versus $79.1 \%$, OR 0.84 (95\% CI 0.79-0.90), $\mathrm{p}<0.001$ and $38.5 \%$ versus $44.7 \%$, OR 0.78 (95\% CI 0.73-0.83), $\mathrm{p}<0.001$, respectively).

Drug resistance rates were markedly lower in the elderly for any drug resistance (6.5 versus $13.9 \%$, OR 0.43 (95\% CI $0.39-0.48)$, $\mathrm{p}<0.001)$ and for multidrug resistance $(0.6$ versus $3.1 \%$, OR 0.18 $(95 \%$ CI $0.13-0.25), p<0.001)$. Drug resistance rates in the foreignborn elderly exceeded those among German-born patients by a factor of 2-3, although the discrepancy was less pronounced than in younger adults. However, the pyrazinamide resistance rate was higher in the elderly (3.2 versus $2.6 \%$, OR $1.28(95 \% \mathrm{CI}$ $1.07-1.52), p=0.006)$ and more frequent in the German-born elderly than in the foreign-born elderly $(3.8 \%(n=188)$ versus $1.7 \%(n=27))$.

Fewer elderly patients than younger patients were diagnosed via active case finding (5.9 versus $18.4 \%$, OR 0.28 (95\% CI $0.25-$ $0.31), \mathrm{p}<0.001)$, including contact tracing (1.6 versus $6.9 \%$, OR $0.21(95 \%$ CI $0.18-0.26))$. TB as a post mortem diagnosis was 


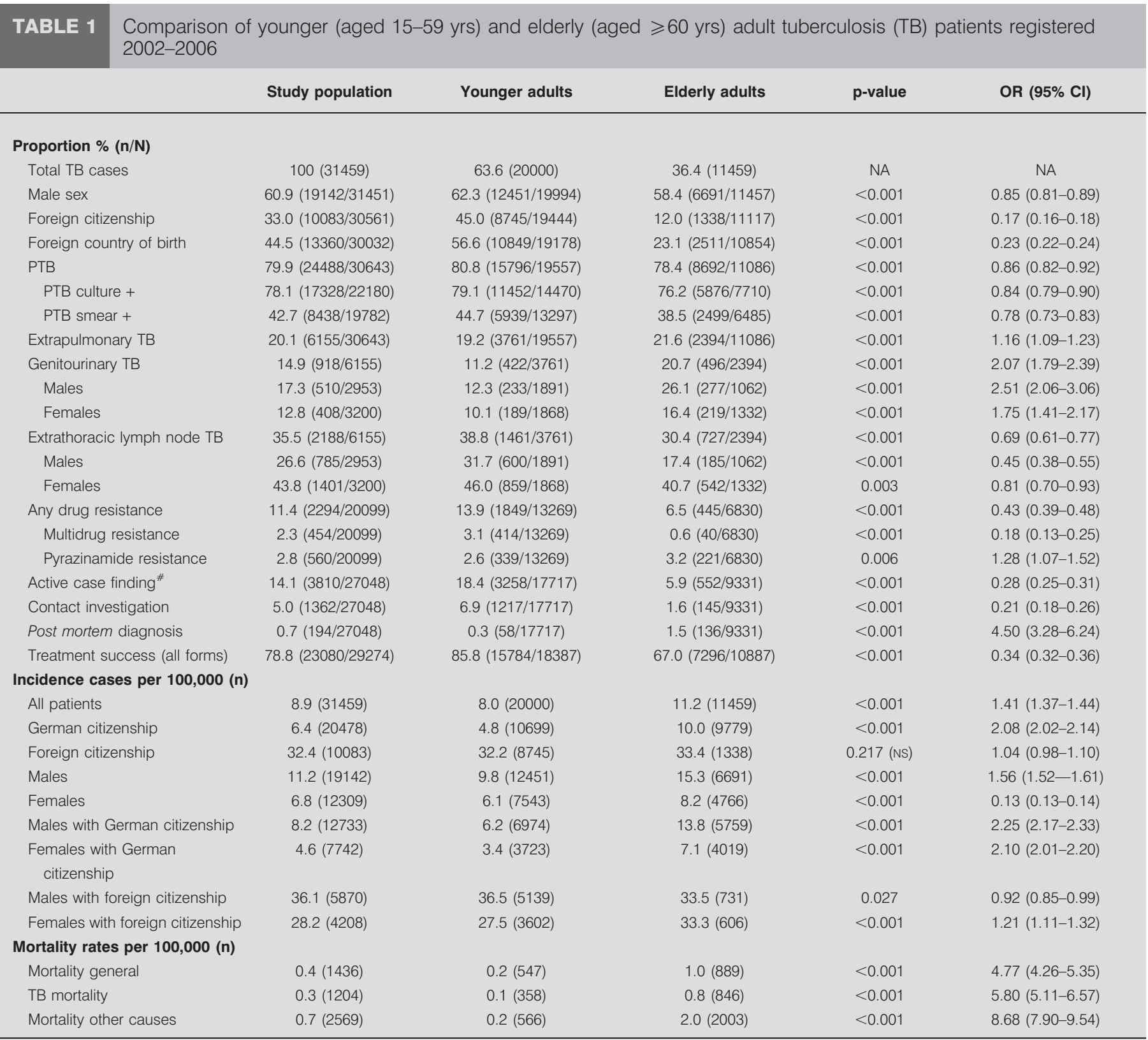

PTB: pulmonary TB; NA: not applicable; NS: not significant. " : active screening of high-risk population groups is defined by the German Protection Against Infection Act as contact investigation or investigation prior to admission to a shared accommodation such as a nursing home or a shelter for homeless people.

more frequently established among elderly patients $(1.5 \%$ versus $0.3 \%$, OR 4.50 (95\% CI 3.28-6.24), p<0.001).

The treatment success rate was lower in the elderly (67.0 versus $85.8 \%$, OR 0.34 (95\% CI 0.32-0.36), p<0.001), dropping steadily with age to $36.8 \%$ in patients aged $\geqslant 90 \mathrm{yrs}$.

Foreign citizenship was associated with a 3.6-fold increase in TB incidence compared with German citizens (32.4 versus 6.4 out of 100,000 for the entire study population). TB incidence peaked in foreign citizens in the 75-79 yrs age group of (45.5 out of 100,000), and in Germans in the $85-89$ yrs age group (14.3 out of 100,000). Among German citizens, TB incidence was two-fold higher in the elderly compared to younger adults
(10.0 versus 4.8 per 100,000 , OR 2.08 (95\% CI 2.02-2.14), $\mathrm{p}<0.001$ ), while no significant age-associated difference was observed among foreign citizens. Among German citizens, TB incidence in males was nearly twice as high as in females regardless of age, while among foreign citizens, sex-associated difference was identified only in younger adults, related to the finding that higher TB incidence was documented in elderly females than in younger females (33.3 versus 27.5 per 100,000), while the converse was true in males (33.5 in elderly verus 36.5 per 100,000 in younger adults).

General mortality in the elderly was remarkably higher than in younger TB cases (1.0 versus 0.2 per 100,000, OR 4.77 (95\% CI $4.26-$ $5.35), \mathrm{p}<0.001$ ), and increased continuously with age ( $\geqslant 90$ yrs: 
2.8 per 100,000$)$. In the elderly age group, male mortality was higher than female $(1.2$ versus 0.7 per 100,000$)$ and foreign citizens had a higher mortality rate than German citizens (1.4 versus 0.9 per 100,000). The fraction of all deaths that were TB-related was smaller in the elderly than in younger adults (29.7 versus $38.7 \%$; $\mathrm{p}<0.001)$.

To our knowledge, this is the first study comparing demographic, clinical and bacteriological characteristics between elderly and younger TB patients among all TB cases notified in Germany in recent years. The study identified several specific epidemiological features of TB in the elderly, such as a high proportion of genitourinary TB (predominantly among males), a considerable fraction of culture-confirmed TB cases, a relatively small contribution of active case finding, comparatively low drug-resistance rates except for pyrazinamide, low treatment success and high mortality rates.

These findings have an impact on the diagnosis of TB in elderly individuals. EPTB is generally difficult to diagnose in this age group due to pre-existing comorbidities [3, 4]. The clinical picture of genitourinary TB in particular may be influenced by pre-existing factors such as prostatic hypertrophy or permanent indwelling bladder catheters, which compromise patients' ability to resolve bacterial infection [4].

The rate of culture-confirmed TB in the elderly (76.2\%) exceeded findings of other studies by $8-15 \%[5,6]$. This may indicate either good or more invasive diagnostic practice in Germany, or it could be explained by patients' or diagnostic delay, resulting in more advanced disease. The latter hypothesis is supported by STORLA et al. [7], who found old age to be a risk factor for diagnostic delay, with factors such as poverty, social isolation, and specific health-seeking behaviour hampering the elderly in gaining timely access to medical care. Regardless of the underlying causes, the subgroup of the elderly have a potential for transmitting TB that may be underestimated.

The low fraction of elderly TB patients identified by active case finding may reflect a high proportion of reactivated TB as well as a low performance of contact tracing. The use of molecular epidemiological methods could help in understanding whether ongoing transmission plays a relevant role [8]. The low contribution of post mortem diagnosis to TB case finding is influenced by a generally low autopsy rate in Germany of 3.1\% (in 1999) [9].

Comparatively low drug-resistance rates in the German elderly support the assumption of a high proportion of reactivated infections acquired prior to introduction of anti-tuberculous antibiotics [3], when TB prevalence was exceptionally high. The higher frequency of pyrazinamide resistance in the elderly may be due to Mycobacterium bovis infections: most of the 164 $M$. bovis TB cases registered in 2004-2006 (no earlier data available) were found in the elderly. Yet strains were not differentiated in all cases, which would be recommendable to avoid unnecessary exposure to the potentially hepatotoxic pyrazinamide [6].

The low treatment success rate and high mortality in TB patients of very old age confirmed earlier findings $[5,10]$. New evidence was provided for a higher mortality rate in males, consistent with the findings of LEFEBVRE and FALZON [10], and in the elderly with foreign citizenship compared to the German elderly. Even among the elderly, almost $30 \%$ of deaths were TB-related. The cause of death can be difficult to determine, as TB may not be the only life-threatening disease and autopsies are rarely performed [9]. Detailed data on underlying diseases, diagnostic delays and course of treatment are therefore needed.

Overall, TB incidence increases with advancing age. Although decreasing infection prevalence can be assumed in successive elderly indigenous cohorts, with rising longevity TB is likely to remain a problem in this population. Despite a high incidence in elderly foreign citizens, absolute case numbers were low, as this was a small population subgroup, presumably due to resettling in the country of origin or a lower life expectancy, both factors which are expected to change over time.

As our analysis is based on notification data, we faced some limiting factors such as potential under-reporting in some patient subgroups (e.g. undocumented migrants), the influence of dynamics of the population structure and migration patterns, lack of clinical insight, e.g. information on specific specimen type (spontaneous, induced or post-bronchoscopic sputum), or details on prior treatment.

Altogether, the present study provides substantial new evidence that elderly adults present a vulnerable group for $\mathrm{TB}$, and stresses the importance of TB as a differential diagnosis in geriatrics to reduce avoidable mortality and prevent further spread of the disease.

\section{B. Hauer*, B. Brodhun*, D. Altmann*, L. Fiebig*, R. Loddenkemper ${ }^{\#}$ and W. Haas*}

*Dept for Infectious Disease Epidemiology, Respiratory Infections Unit, Robert Koch Institute, and ${ }^{\#}$ German Central Committee Against Tuberculosis, Berlin, Germany.

Correspondence: B. Hauer, Dept for Infectious Disease Epidemiology, Respiratory Infections Unit, Robert Koch Institute, DGZ-Ring 1, 13086 Berlin, Germany. E-mail: HauerB@rki.de

Statement of Interest: None declared.

Acknowledgements: We thank the German Ministry of Health for kindly supporting this analysis.

\section{REFERENCES}

1 Robert Koch-Institut. Bericht zur Epidemiologie der Tuberkulose in Deutschland 2008. www.rki.de/cln_178/nn_274324/DE/Content/ InfAZ/T/Tuberkulose/Download/TB2008.html Date last accessed: May 18, 2011; date last updated: March 22, 2010.

2 Dutt AK, Stead WW. Tuberculosis in the elderly. Tuberculosis 1993; 77: 1353-1368.

3 Davies PDO. Tuberculosis in the elderly. Epidemiology and optimal management. Drugs Aging 1996; 8: 436-444.

4 Owen RE, Allen DM. Infections in the elderly. Singapore Med J 1991; 32: 179-182.

5 Teale C, Goldman M, Pearson SB. The association of age with the presentation and outcome of tuberculosis: a five-year survey. Age Ageing 1993; 22: 289-293.

6 Lee JH, Han DH, Song JW, et al. Diagnostic and therapeutic problems of pulmonary tuberculosis in elderly patients. $J$ Korean Med Sci 2005; 20: 784-789. 
7 Storla DG, Yimer S, Bjune GA. A systematic review of delay in the diagnosis and treatment of tuberculosis. BMC Public Health 2008; 8: 15.

8 Borgdorff MW, van der Werf MJ, de Haas PEW, et al. Tuberculosis elimination in the Netherlands. Emerg Infect Dis 2005; 11: 597-602.

9 Brinkmann B, Du Chesne A, Vennemann B. A survey of autopsy rates in Germany. Dtsch Med Wochenschr 2002; 127: 791-795.
10 Lefebvre N, Falzon D. Risk factors for death among tuberculosis cases: analysis of European surveillance data. Eur Respir J 2008; 31: $1256-1260$.

\section{Rituximab in bronchiolitis obliterans after haematopoietic stem cell transplantation}

\section{To the Editors:}

Bronchiolitis obliterans (BO) following allogeneic haematopoietic stem cell transplantation (HSCT) is recognised as a pulmonary manifestation of chronic graft-versus-host disease (GVHD) [1, 2]. As lung biopsy is rarely performed in these patients, the diagnosis of BO syndrome (BOS) usually relies on pulmonary function testing (PFT) and computed tomography (CT) lung scans [1, 2]. Very little retrospective data exist regarding the treatment of BOS, which currently consists of increasing a patient's baseline dose of systemic immunosuppressive therapy; however, this treatment strategy has been shown to have poor efficacy [2,3]. The efficacy of new drugs for the treatment of chronic GVHD, including the anti-CD20 monoclonal antibody rituximab, must be specifically evaluated for BOS. Here, we report three cases in which patients received rituximab for $\mathrm{BO}$ after allogeneic HSCT.

Patient 1 was a 15-yr-old male who received bone marrow from a sibling human leukocyte antigen (HLA)-matched donor for acute myeloid leukaemia after a myeloablative conditioning regimen that included 12 Gy fractionated total body irradiation (FTBI) and cyclophosphamide. 5 months after transplantation, he was diagnosed with chronic liver GVHD that led to the initiation of prednisone at a dosage of $1 \mathrm{mg} \cdot \mathrm{kg}^{-1} \cdot \mathrm{day}^{-1}$ as well as tacrolimus. 8 months after transplantation, as his liver GVHD was coming under control, he presented with dyspnoea, dry cough and chest pain. At this time, he was receiving tacrolimus and prednisone at a dosage of $20 \mathrm{mg} \cdot \mathrm{day}^{-1}$. PFT revealed a severe obstructive pattern based on a forced expiratory volume in $1 \mathrm{~s}$ (FEV1) of $1.13 \mathrm{~L}$ (33\% predicted), a forced vital capacity (FVC) of $2.79 \mathrm{~L}$ (65\% pred) and FEV1/FVC ratio of $41 \%$. A lung CT scan demonstrated the presence of air trapping and a pneumomediastinum. A pulmonary infection was ruled out, and the diagnosis of post-allogeneic HSCT BOS was made. The patient presented with no other signs of chronic GVHD at this time. The prednisone dosage was increased to $2 \mathrm{mg} \cdot \mathrm{kg}^{-1} \cdot \mathrm{day}^{-1}$, tacrolimus treatment was continued, and mycophenolate mofetil, inhaled steroids, bronchodilators and low-dose macrolides were added to the treatment regimen. Despite this treatment and although the pneumomediastinum resolved, PFT revealed consistent deterioration over the following months. $4 \mathrm{yrs}$ after transplantation, the patient was still receiving $20 \mathrm{mg} \cdot$ day $^{-1}$ prednisone, inhaled steroids, bronchodilators and low-dose macrolides, and developed a left spontaneous pneumothorax that necessitated pleurectomy. A surgical lung biopsy was simultaneously performed. A histological examination confirmed the diagnosis of $\mathrm{BO}$ : several bronchioles were narrowed or completely obliterated by fibrosis, while others had walls containing lymphocytes, primarily B-cells (fig. 1). In response to these findings, the patient received two infusions
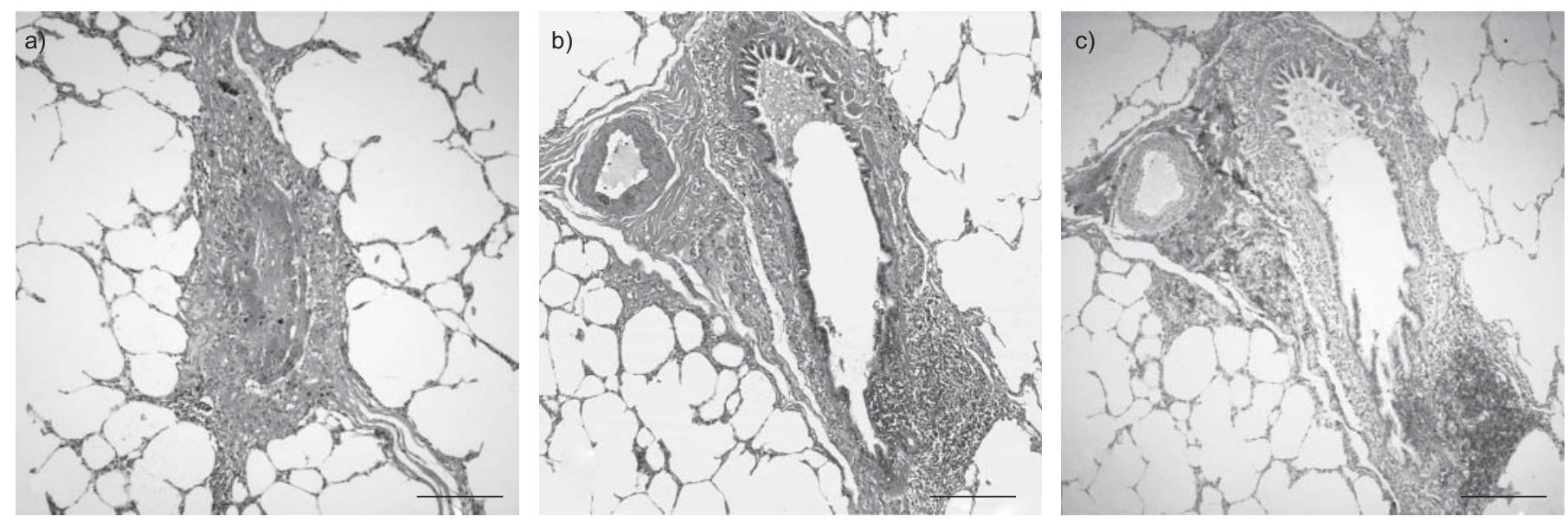

FIGURE 1. a) The lumen of this bronchiole is completely obliterated by dense fibrosis (haematoxylin and eosin staining (HES)). b) A nodular lymphoid infiltrate is found in the wall of this bronchiole (HES). c) The lymphocytes of the infiltrate are predominantly B-cells (immunohistochemistry with anti-CD20 antibody). Scale bars $=200 \mu \mathrm{m}$. 